\title{
ARTICLE OPEN Wireless organic electronic ion pumps driven by photovoltaics
}

\author{
Marie Jakešová (D) ${ }^{1}$, Theresia Arbring Sjöström¹, Vedran Đerek ${ }^{1,2}$, David Poxson ${ }^{1}$, Magnus Berggren ${ }^{1}$, Eric Daniel Głowacki $\mathbb{D}^{1,2}$ and \\ Daniel T. Simon (D) \\ The organic electronic ion pump (OEIP) is an emerging bioelectronic technology for on-demand and local delivery of \\ pharmacologically active species, especially targeting alkali ions, and neurotransmitters. While electrical control is advantageous for \\ providing precise spatial, temporal, and quantitative delivery, traditionally, it necessitates wiring. This complicates implantation. \\ Herein, we demonstrate integration of an OEIP with a photovoltaic driver on a flexible carrier, which can be addressed by red light \\ within the tissue transparency window. Organic thin-film bilayer photovoltaic pixels are arranged in series and/or vertical tandem to \\ provide the 2.5-4.5 V necessary for operating the high-resistance electrophoretic ion pumps. We demonstrate light-stimulated \\ transport of cations, ranging in size from protons to acetylcholine. The device, laminated on top of the skin, can easily be driven \\ with a red LED emitting through a $1.5-\mathrm{cm}$-thick finger. The end result of our work is a thin and flexible integrated wireless device \\ platform.
}

npj Flexible Electronics (2019)3:14; https://doi.org/10.1038/s41528-019-0060-6

\section{INTRODUCTION}

The field of bioelectronic medicine is presently in a period of rapid growth. ${ }^{1,2}$ A large motivation exists to develop smart electronic solutions for targeted and precisely metered dosing of drugs. ${ }^{3}$ The ability to interface delivery devices together with biosensors allows an internet-of-things feedback control of drug delivery with real-time data monitoring, logging, and decision making. ${ }^{4}$ Flexible and imperceptible electronics concepts are key ingredients of this new bioelectronics device design. ${ }^{5}$ One of the emerging devices for direct electrically controlled drug delivery is the organic electronic ion pump (OEIP). This device allows precise electronicionic transduction, resulting in convection-free delivery of smallsized ionic species. ${ }^{6,7}$ OEIPs operate on the principle of selective electrophoretic ion transport from a source solution to a target solution across an ion exchange membrane (IEM). The latter is fabricated from ion-conducting polyelectrolytes, which are polymers with a high density of specific charge. Based on the polarity of the charged side groups, we distinguish cation (negatively charged polymer) and anion (positively charged polymer) exchange membranes. By applying an electric field along the membrane, the specific ions are transported from one end (source) to the other (target) with a negligible amount of solvent being co-transported with the ions. ${ }^{7}$ The technology has been deployed successfully in several in vivo applications from sensory modulation $^{8}$ to termination of epileptiform spiking. ${ }^{9}$ Drug release can be controlled via bluetooth. ${ }^{10}$ All of the demonstrated in vivo applications require a power supply along with a proper controller unit that are wired through the tissue to reach the OEIP device. The issue of wiring represents a hurdle for applying this technology in biomedical settings. Wiring is invasive and increases the chances of inflammation, especially for long-term therapy applications. The motivation of developing implants with a minimal mechanical footprint, which maximizes surgical simplicity and patient comfort, encourages the search for wireless solutions.
Several approaches are currently explored for wireless power delivery and transduction to implanted devices. So far, these include batteries, body-energy harvesting (e.g., bio-fuel cells, thermoelectric, or piezoelectric generators ${ }^{11}$ ) and external power units transmitting energy in (ultrasonic devices, inductive power transmission, and light stimulation). ${ }^{12}$ Photoconversion using photovoltaic (PV) devices has been demonstrated to power various epidermal ${ }^{13}$ and implantable technologies. ${ }^{14}$ Rigid monocrystalline silicon PV modules have been used to power cardiac pacemakers in a porcine model. ${ }^{15,16}$ Flexible GalnP/GaAs PVs were able to support pacemaker operation in a hairless mouse model. ${ }^{17}$ Recently, Rogers et al. reported biodegradable PV arrays based on polycrystalline silicon. ${ }^{18}$

The starting question of this work is how to fabricate an integrated wireless OEIP, which retains the low thickness and flexibility intrinsic to the organic electronic components constituting the OEIP itself. As an electrophoretic device, a precise voltage needs to be delivered in order to drive a specific ionic current, and thus a specific rate of delivery. Moreover, IEMs typically have a high resistivity to provide the necessary ion selectivity and good ON/OFF ratio, therefore OEIPs require relatively high voltages but low currents. To satisfy these parameters, we chose to couple the OEIP with thin organic photovoltaic (OPV) cells, which can easily be arranged in series on a flexible substrate in order to deliver the voltage necessary to drive electrophoretic ion transport under low light-intensity conditions. The OPV active material was chosen to strongly absorb red light in the tissue transparency window $(600-700 \mathrm{~nm})$. OPVs, by virtue of their high absorption coefficient materials, allow fabrication of devices with the active layers being $<300 \mathrm{~nm}$, thereby providing flexibility and minimizing the overall implant thickness. We demonstrate a flexible, integrated, and light-driven ion pump technology, which enables desired delivery operation with low-intensity levels of red light. We validate its performance and stability with proton and acetylcholine (ACh)

\footnotetext{
${ }^{1}$ Laboratory of Organic Electronics, ITN Campus Norrköping, Linköping University, SE-60174 Norrköping, Sweden and ${ }^{2}$ Wallenberg Centre for Molecular Medicine, Linköping University, SE-58185 Linköping, Sweden

Correspondence: Marie Jakešová (marie.jakesova@liu.se) or Eric Daniel Głowacki (eric.glowacki@liu.se)
}

Received: 11 March 2019 Accepted: 15 July 2019

Published online: 30 July 2019 

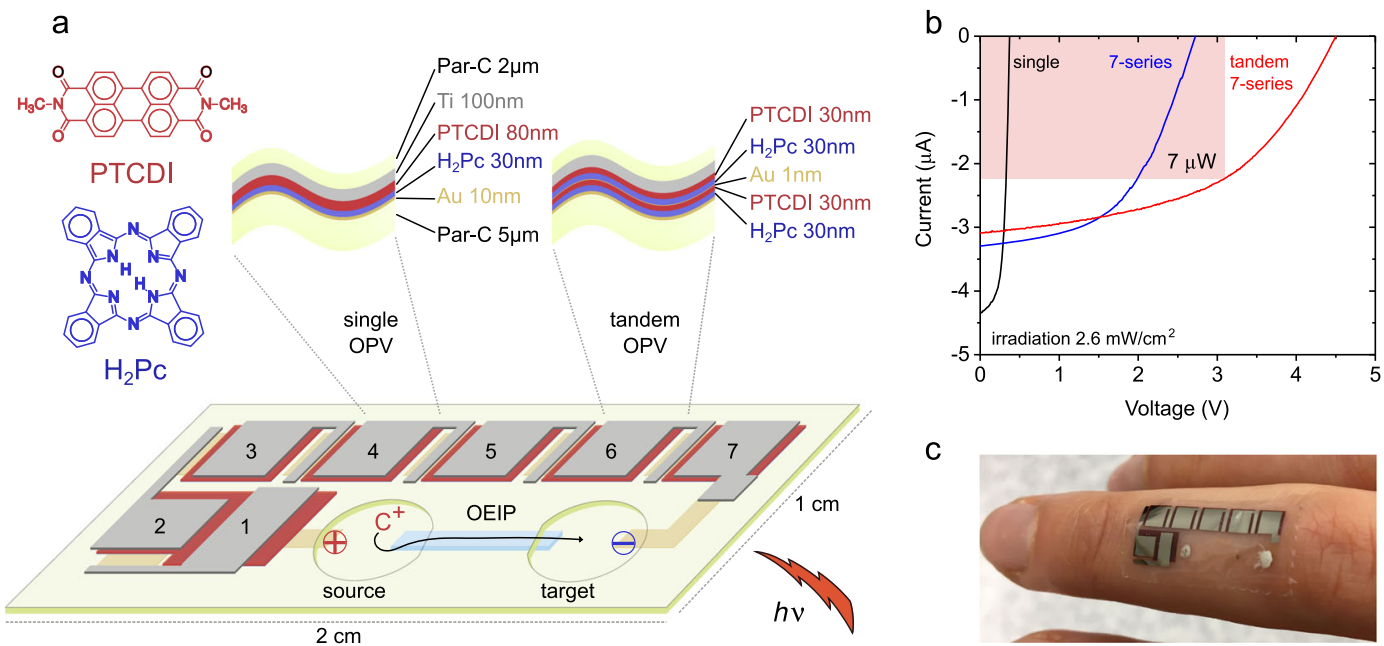

C

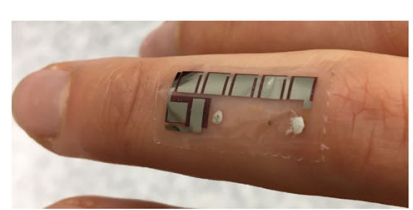

Fig. 1 Flexible organic electronic ion pumps integrated with organic photovoltaics. a Schematics of the integrated OPV and OEIP components on thin parylene-C substrates. b I $(V)$ curves measured for a single diode and a sevenfold series of single and tandem diodes under diffuse illumination of $2.6 \mathrm{~mW} / \mathrm{cm}^{2}$ at $626 \mathrm{~nm}$. c The integrated device adhered on the skin.

transport as examples, and perform a demonstration of the device operating with illumination through $1.5 \mathrm{~cm}$ of tissue and bone.

\section{RESULTS AND DISCUSSION}

The integrated OPV-OEIP components are illustrated in Fig. 1a. To assure low invasiveness, durability, biocompatibility, and flexibility we chose a thin parylene-C film as the device substrate. ${ }^{19}$ Parylene- $C$ with a thickness of $5 \mu \mathrm{m}$ was readily processed on $4^{\prime \prime}$ glass wafers using the conventional CVD process. ${ }^{20}$ The photovoltaic material combination was dictated by the necessary operation within the red/near-infrared tissue transparency window and preferably should have good indications of biocompatibility. The materials of choice were metal-free phthalocyanine $\left(\mathrm{H}_{2} \mathrm{Pc}\right)$ and $N, N^{\prime}$-dimethylperylene-3,4,9,10-tetracarboxylic diimide (PTCDI). These small molecules can be processed by vapor sublimation and easily patterned by stencil masking. This donor-acceptor stack has been used for photocapacitor devices, ${ }^{21}$ which were stable in physiological media, in cell culture, and appears to have promising biocompatibility and stability. Since, by design, the OEIP is a highly resistive device, the OPV operating voltages have to be maximized. A single $\mathrm{H}_{2} \mathrm{Pc}$ /PTCDI bilayer using semitransparent $\mathrm{Au}$ as a bottom electrode and Ti as top contact delivers roughly a maximum of $400 \mathrm{mV}$ under illumination. For lateral OEIPs (ion transport parallel to plane of substrate), driving of smaller cations such as protons or potassium ions results in an OEIP channel resistance typically in the range of $1-10 \mathrm{M} \Omega$, while larger ions such as $\mathrm{ACh}$ give $\sim 100 \mathrm{M} \Omega$. Therefore, to drive therapeutically relevant currents of $0.01-1 \mu \mathrm{A}$, several volts are necessary. ${ }^{7}$ We realized this by connecting seven individual OPV cells in series. To be able to increase the voltage further, we also fabricated the tandem version of the series devices, achieving output in the range of $2.8-4.5 \mathrm{~V}$. The integrated device was built sequentially starting with the OEIP. Firstly, the cation exchange membrane devices were patterned via lithography and reactive ion etching (RIE). To connect the OEIP with the OPV series, a suitable common bottom contact must be chosen. We initially explored indium tin oxide, which despite other advantages turned out to be a poor choice due to electrochemical instability during OEIP operation. We found that thin evaporated gold $(10 \mathrm{~nm})$ had excellent adhesion to the parylene- $C$ and combined good conductivity, transparency, and electrochemical stability. The thin semitransparent gold electrodes were defined by photolithographic processing using chemical wet etching. The OPV organic layer $\left(\mathrm{H}_{2} \mathrm{Pc} / \mathrm{PTCDI}\right)$ and top titanium electrodes were deposited via thermal evaporation through stencil masks. Tandem devices consisted of two $\mathrm{H}_{2} \mathrm{Pc} / \mathrm{PTCDI}$ bilayers evaporated sequentially with a thin interlayer of $\mathrm{Au}$ in between. Finally, the devices were encapsulated with a second layer of parylene-C $(2 \mu \mathrm{m})$ with lithographically defined openings for the source and target electrolytes.

The integrated devices could be probed to gauge the performance of the OPV components (Fig. 1b). We focused on optimizing the devices in the context of levels of low-intensity diffuse red light as would be expected at the depth of $\sim 1 \mathrm{~cm}$ of tissue (i.e., a few $\mathrm{mW} / \mathrm{cm}^{2}$ ). A single OPV cell has an open circuit voltage $\left(\mathrm{V}_{\mathrm{OC}}\right)$ of $0.4 \mathrm{~V}$ and $100 \mu \mathrm{A} / \mathrm{cm}^{2}$ short-circuit current density $\left(\mathrm{J}_{\mathrm{SC}}\right)$ when using a high-density light emitting diode (LED) array with diffusor $\left(626 \mathrm{~nm}\right.$, intensity $\left.2.6 \mathrm{~mW} / \mathrm{cm}^{2}\right)$. Under the same condition, the series connection of seven diodes delivers $V_{O C} 2.7 \pm$ $0.1 \mathrm{~V}$, short-circuit current $\left(\mathrm{I}_{\mathrm{SC}}\right)$ of $2.8 \pm 0.4 \mu \mathrm{A}$, and a total output power (maximum power point, MPP) of $3.7 \pm 0.9 \mu \mathrm{W}$ (mean \pm SD, 34 devices). The series of tandem devices has $\mathrm{V}_{\mathrm{OC}} 4.4 \pm 0.2 \mathrm{~V}, \mathrm{I}_{\mathrm{SC}}$ $2.0 \pm 0.6 \mu \mathrm{A}$, and MPP $4.3 \pm 1.6 \mu \mathrm{W}$ (mean \pm SD, 24 devices). The fabrication process yields $95 \%$ of the single OPV, $85 \%$ of the tandem OPV ( $n=56$ each), and 94\% of the OEIP devices functional. We find essentially identical performance when driving with LEDs of 660,680 , or $720 \mathrm{~nm}$ emission as well (Supplementary Fig. 1), indicating that various commercial-LED wavelengths within the tissue transparency window can serve to drive the device. These parameters remain constant after the devices are peeled off the glass support (Supplementary Fig. 2). An image of the peeledoff device laminated on skin is shown in Fig. 1c.

Once we established the performance and stability of the seriesconnected OPVs, we verified the functionality of the OEIPs as well (see the 'Methods' section and Supplementary Fig. 3). ${ }^{7,22}$ Having determined that the fabrication process yields stable and reproducible OPV and OEIP components, we moved on to test wireless light-driven actuation of the OEIPs. As a simple wire-free readout, we tested the pumping of protons from the source electrolyte, containing $\mathrm{HCl}$, to a target, containing a methyl red (MR) $\mathrm{pH}$ indicator. A set of $\mathrm{H}^{+}$-loaded devices were placed in a humidity chamber on a high-density LED array and photographs were taken between 0 and $10 \mathrm{~min}$ (Fig. 2a). The target electrolytes gradually turned from yellow to red, with a $\mathrm{pH}$ gradient (e.g., at $3 \mathrm{~min}$ ) across the target electrolyte, demonstrating the expected proton delivery. The process was indeed dominated by electrophoretic migration and not heat-enhanced diffusion, because control devices without a connected OPV module showed no color change, thus the resistance of the IEM was high enough to 

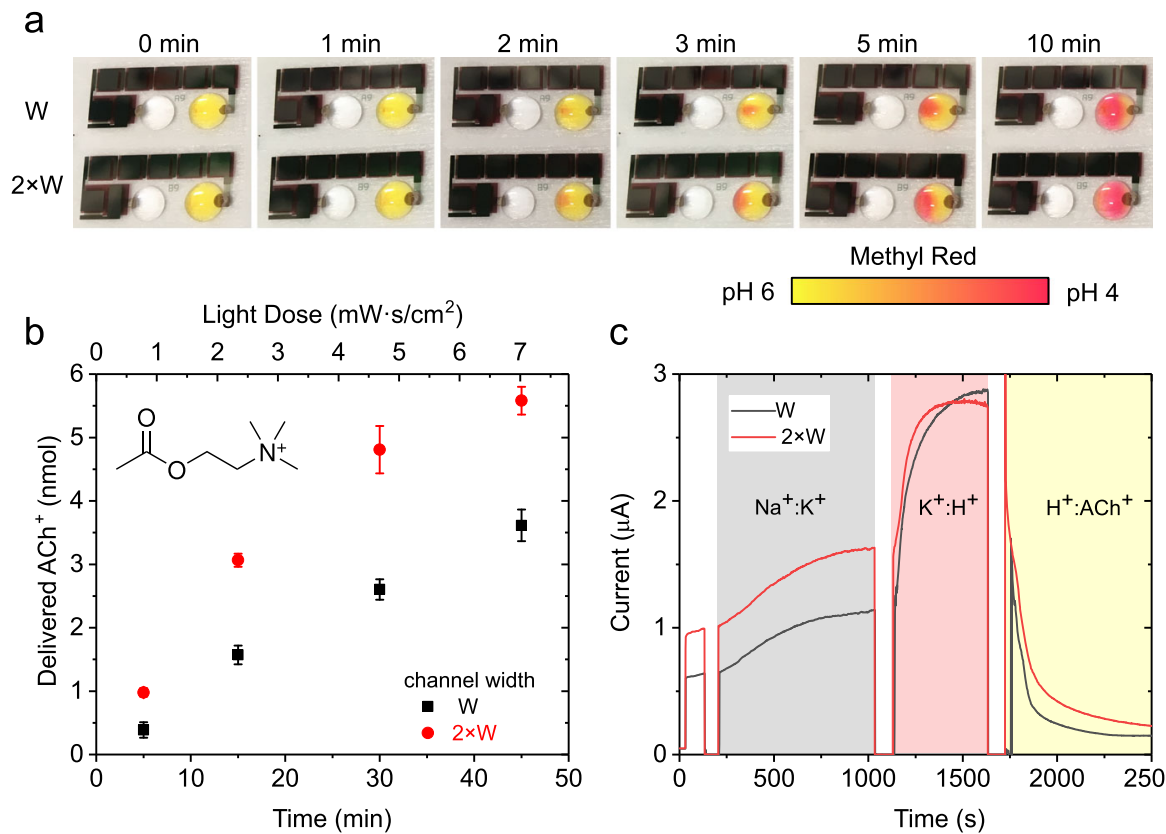

C

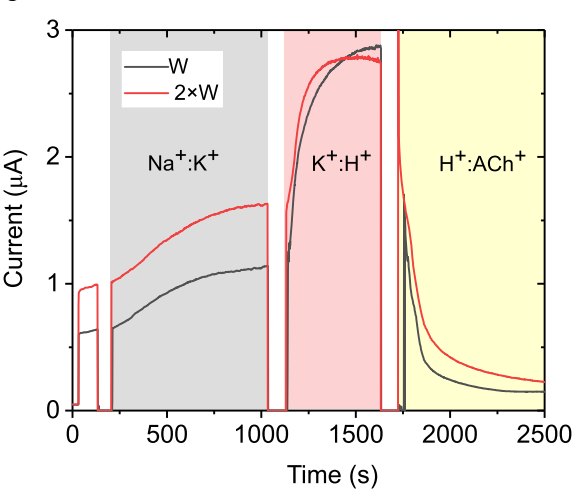

Fig. 2 Light-driven ion pump operation. a Photodriven proton-pumping experiment validated with methyl red pH indicator dye. The source reservoirs are positioned on the left, and contain $0.1 \mathrm{M} \mathrm{HCl}$, while the target reservoirs on the right have methyl red. This dye is initially yellow but turns red with lowering $\mathrm{pH}$. The ion delivery point (end of ion channel) is at the leftmost point of the target reservoir, thus the $\Delta \mathrm{pH}$ starts there. b Acetylcholine pumping experiment, demonstrating effective OPV driven ion transport of a larger ion using the tandem series devices. Error bars = s.d. c Online current experiment on the photodriven ion pumps of the two different sizes. The ions in the source electrolyte are switched from $\mathrm{Na}^{+}$to $\mathrm{K}^{+}$to $\mathrm{H}^{+}$and finally $\mathrm{ACh}^{+}$. The current changes according to the ion's conductivity. The mode of OPV operation is voltage-limited in case of the larger ions $\mathrm{ACh}^{+}, \mathrm{Na}^{+}$, and $\mathrm{K}^{+}$, but current-limited for $\mathrm{H}^{+}$. This is evident by similar levels of $\mathrm{H}^{+}$current for the devices of the two different OEIP geometries.

prevent passive diffusion. This $\mathrm{pH}$ indicator experiment is a convenient way to simultaneously test many devices in parallel and to qualitatively prove that they truly work (Supplementary Fig. 4). In order to quantitatively validate the devices and to verify the possibility of transporting larger ions, we next studied the lightdriven transport of $\mathrm{ACh}$, a cationic neurotransmitter previously used in several practical OEIP applications. ${ }^{23}$ The tandem series OPV devices were used for the ACh experiments, due to the lower conductivity of ACh. ACh chloride was used in the source electrolyte. The target electrolyte was collected after exposure to a known light dose, and the concentration of delivered ACh was measured using an enzymatic assay. The amount of $\mathrm{ACh}^{+}$ delivered was proportional to the light dose applied, providing evidence for light-to-electric field-driven transport (Fig. 2b, average of three devices). In order to determine the pumping efficiency (ratio of ion transported to electrical current through the device ${ }^{24}$ ), we fabricated devices allowing us to measure the circuit current during the experiment (Supplementary Fig. 5). The efficiency was $73 \pm 5 \%(n=4)$, suggesting cotransport of $\mathrm{H}^{+}$ions present in the $\mathrm{AChCl}$ solution. The same type of device was used for online monitoring of the ion exchange within the IEM (Fig. 2c). This experiment reveals different regimes of the integrated device operation. The experiment starts with the ion channel loaded with $\mathrm{Na}^{+}$. Then the source electrolyte is exchanged for higher conductivity $\mathrm{K}^{+}$ions. At both of these points, the transport is voltage-limited. However, once the source electrolyte is switched to the highest conductivity $\mathrm{H}^{+}$, the transport becomes currentlimited, with the OPV not being able to deliver current higher than close to $\mathrm{I}_{\mathrm{sc}}$. Finally, when $\mathrm{H}^{+}$is exchanged with the low conductivity $\mathrm{ACh}^{+}$, the transport is once more voltage-limited. This is further evidenced by measuring devices with different IEM geometry. To evaluate stability of the integrated devices, we measured the OPV parameters after fabrication, and following 15 and 72 (34 for tandems) days of storage in phosphate buffered saline (PBS). The OEIP components were independently tested and no degradation was found. The OPV performance of the devices stored in PBS declined slightly over time, however, all devices remained functional, and worked for proton pumping (Supplementary Fig. 6). For series of single devices, the value of $V_{O C}$ remains constant while $I_{S C}$ and MPP are the parameters undergoing gradual degradation, indicating that the series resistance of the device is increasing over time. Similarly, the tandem devices also show gradual decrease in the $I_{S C}$ and MPP. However, the $V_{O C}$ of the tandem devices is also affected, suggesting that the tandem stack is not fully stable after a month of storage in PBS. To evaluate flexibility of our integrated devices we performed a preliminary bending test by wrapping our devices around syringe needles of 1.2 and $0.6 \mathrm{~mm}$ diameter (Supplementary Fig. 7). The devices survived multiple cycles of bending over the larger diameter needle, while bending over the smaller diameter needle generally resulted in partial failure after a few bending cycles. Crumbling leaves half of the single-devices shorted. To produce devices with more extreme bending tolerances the substrate and encapsulating layers should be optimized to ensure that the sensitive device areas lie within the neutral mechanical plane. ${ }^{5}$

Finally, to put the wireless operation of the OEIP into context, we attempted through-tissue operation. A single device was placed on a finger $(\approx 1.5 \mathrm{~cm}$ diameter) and was illuminated with a $3 \mathrm{~W}, 630 \mathrm{~nm}$ LED from below the finger (Fig. 3a). We established that a light intensity of $\sim 2 \mathrm{~mW} / \mathrm{cm}^{2}$ penetrated through the finger by placing a calibrated silicon diode at the skin surface. An I(V) curve measured while illuminating through the finger showed similar behavior as while illuminating the OPV series with $2.6 \mathrm{~mW} /$ $\mathrm{cm}^{2}$, therefore the estimated light intensity is reasonable (Fig. $3 \mathrm{~b}$ ). The experimenter held her hand over the light source for $3 \mathrm{~min}$, and the $\mathrm{H}^{+}$transport was verified with the use of the $\mathrm{pH}$ indicator (Fig. 3c). Subjectively, though the light source was warm, it was not uncomfortably hot and the measurement could be easily performed. This experiment demonstrates that a simple 
a

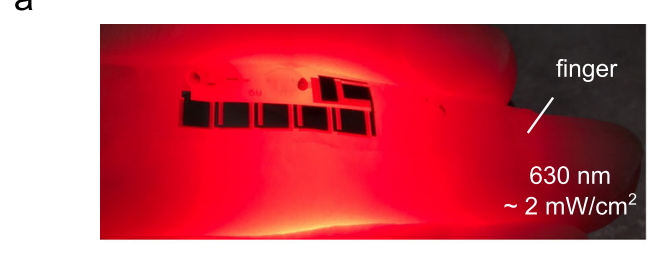

b

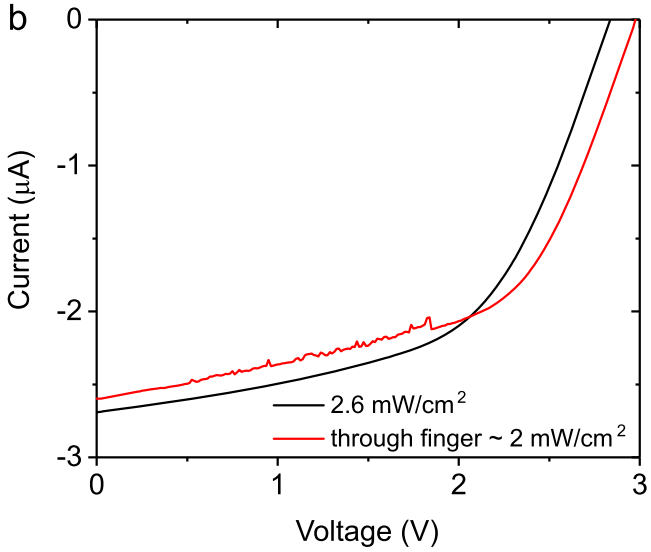

C

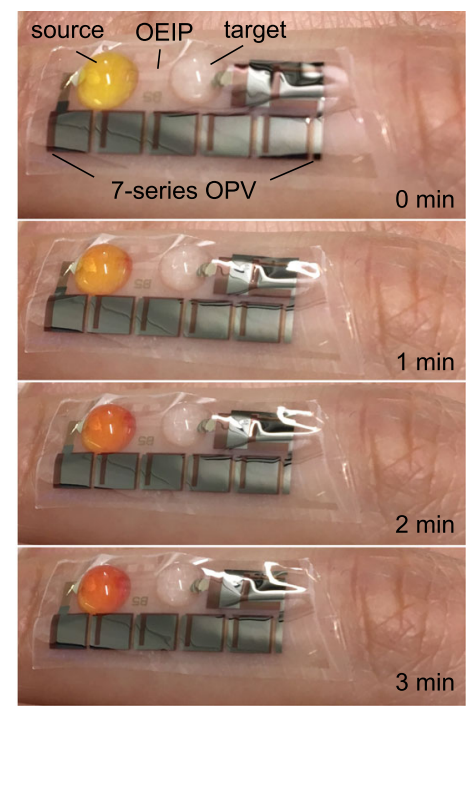

Fig. 3 Through-tissue light actuation of ion transport. a An integrated OEIP with OPV array laminated on the finger of the experimenter, with hand placed over a red LED emitting at $630 \mathrm{~nm}$. A light intensity of around $2 \mathrm{~mW} / \mathrm{cm}^{2}$ penetrates through the finger. $\mathbf{b} \mathrm{I}(V)$ curves measured with the device illuminated through the finger, compared with a measurement registered using a similar light intensity. c Validation of successful light-driven $\mathrm{H}^{+}$pumping. $0.1 \mathrm{M} \mathrm{HCl}$ is added to the reservoir on the right, while the methyl red pH indicator is put in the left side reservoir. The $\mathrm{pH}$ gradient (lower $\mathrm{pH}$ on the right) is faintly observable at $1 \mathrm{~min}$. The red light is shone through the finger and turned off at $1-$ min intervals, at which point photographs were taken.

commercial LED can easily deliver enough power through tissue and bone to actuate the OEIP over a distance of at least $1.5 \mathrm{~cm}$.

In this work, we have endeavored to design a wirelessly controlled OEIP, which leverages the flexibility and low mechanical profile offered by organic electronic materials. As a result, we have shown that small-molecule photovoltaic devices can be readily fabricated into small arrays, connected in series, that deliver the high voltage needed to drive ion pumps, and appear to be stable. Our work demonstrates the efficacy with which these arrays can harvest relatively low-intensity diffuse light in the tissue transparency window. This suggests that this approach may be a suitable strategy for powering other in vivo technologies. Given the current level of demonstrated performance and stability, we believe this platform is on track for enabling wireless OEIP applications in a more realistic biomedical context.

\section{METHODS}

\section{Device fabrication}

4-inch soda lime glass wafers (University Wafer, $550 \mu \mathrm{m}$ thick) were cleaned in a circulating $2 \%$ solution of Hellmanex III detergent heated to $50{ }^{\circ} \mathrm{C}$ for $30 \mathrm{~min}$ followed by a high-pressure rinse with acetone and distilled water (DI). The wafers were then treated in $\mathrm{O}_{2}$ plasma (Diener electronic $\mathrm{GmbH}, 100 \mathrm{~W}, 5 \mathrm{~min}$ ). Immediately after, a 5 - $\mu \mathrm{m}$-thick parylene$\mathrm{C}$ layer was deposited via chemical vapor deposition (Diener electronic $\mathrm{GmbH})$. The parylene surface was then activated with $\mathrm{O}_{2}$ plasma $(50 \mathrm{~W}$, $2 \mathrm{~min}$ ). First, the OEIP part of the device was formed. Poly(4-styrenesulfonic acid-co-maleic acid) (PSS-co-MA, Sigma-Aldrich, $M_{w} \sim 20,000,25 \mathrm{wt} \%$ in $\mathrm{H}_{2} \mathrm{O}$, sequentially dialyzed in $\mathrm{HCl}$ ) $\mathrm{H}^{+}$form (4 wt\% in a mixture of Dl:1propanol, 1:1) was mixed with $1.5 \mathrm{wt} \%$ polyethylene glycol (PEG, $\mathrm{M}_{\mathrm{w}} \sim 400$ ) and 0.2 vol\% (3-glycidyloxypropyl)trimethoxysilane (GOPS). The mixture was mixed and after $15 \mathrm{~min}$ was spin coated at $1500 \mathrm{rpm}$ onto the $4^{\prime \prime}$ substrates. Two layers of the PSS-Co-MA mixture were deposited with a bake step at $110^{\circ} \mathrm{C}$ for at least $1 \mathrm{~h}$ after each (more details on the PSS-coMA fabrication in ref. ${ }^{25}$ ). A thin film of polymethylmethacrylate (PMMA, $M_{w}$ $12,000,4 \mathrm{mg} / \mathrm{mL}$ in diethyl carbonate) was spin coated on the PSS-co-MA layer, followed by Shipley S1813 G2 positive photoresist. The pattern was obtained through exposure by Karl Süss MA6/BA6 mask aligner and development in Microposit MF319 and RIE (150 W, 95 s, O 100 sccm; $\mathrm{CF}_{4}$ $200 \mathrm{sccm}$ ). Then the OPV component followed. A $10 \mathrm{~nm}$ film of Au was thermally deposited over the whole wafer using a physical vapor deposition (PVD) chamber in a vacuum of $<2 \times 10^{-6}$ Torr using a rate of 1-2 Å/s. The gold layer was patterned by lithography using S1805/ Microposit MF319 and KI/I for chemical wet etch. The photoresist was removed in acetone. The PSS-co-MA ion channels were converted from the $\mathrm{H}^{+}$form to the $\mathrm{Na}^{+}$form by immersing the wafer in a $0.1 \mathrm{M} \mathrm{NaCl}$ aqueous solution for $3 \mathrm{~min}$. The wafers were dried in an oven at $110^{\circ} \mathrm{C}$ for $10 \mathrm{~min}$. The organic semiconductor $p-n$ junctions were obtained by PVD through a stencil mask. Metal-free phthalocyanine $\left(\mathrm{H}_{2} \mathrm{Pc}\right.$, Alfa Aesar), and $\mathrm{N}, \mathrm{N}^{\prime}$-dimethylperylene tetracarboxylic diimide (PTCDI, BASF) thin films were evaporated from resistively heated crucibles in a vacuum of $1 \times 10^{-6}$ mbar at a rate of $1-4 \AA / s$ to a thickness of $30 / 80 \mathrm{~nm}$, respectively. The thicknesses were chosen to obtain the best compromise between OPV performance and the device yield. Both materials were previously purified by temperature gradient sublimation three times. In case of the tandem devices, the structure contained two bilayers of $30 / 30 \mathrm{~nm} \mathrm{H}_{2}$ Pc/PTCDI with a $1 \mathrm{~nm}$ Au interlayer, the stencil mask was fixed to the substrate to allow transfer between organic and metal evaporator chambers, the sequence was: organic/metal/organic. A $100 \mathrm{~nm} \mathrm{Ti}$ top electrode was deposited using a stencil mask in a PVD chamber in a vacuum of $<2 \times 10^{-6}$ Torr using a rate of $1-15 \AA / s$. Finally, the devices were encapsulated, and source and target reservoirs of the OEIP were formed. A $2 \mu \mathrm{m}$-thick parylene-C layer was deposited on the substrate with an adhesion promoter (methacryloxypropyltrimethoxysilane, A-174) in situ. An aluminum RIE hard mask was used for opening the source, target, and contact pads. The openings were defined by S1818, a $50 \mathrm{~nm}$ layer of Al was evaporated in a PVD chamber in a vacuum of $<2 \times 10^{-6}$ Torr using a rate of $5-15 \AA /$ s. The metal was then lifted-off in acetone using mild sonication. The encapsulating parylene- $C$ was removed by RIE ( $\left.150 \mathrm{~W}, \mathrm{O}_{2} 400 \mathrm{sccm} ; \mathrm{CF}_{4} 100 \mathrm{sccm}\right)$. Finally, the $\mathrm{Al}$ mask was etched using a commercial wet etch solution. The exposed $\mathrm{Au}$ electrodes were covered by an $\mathrm{Ag} / \mathrm{AgCl}$ paste prior to characterization.

\section{Device characterization}

All electrical characterization was performed using a Keithley 4200-SCS and Keithley 2602A source meter. The performance of the OEIP component was always independently verified by adding electrolyte to the source and target reservoirs, contacting them with $\mathrm{Ag} / \mathrm{AgCl}$ probes, and measuring steady-state ionic currents. To probe the selectivity, the transported ion (in the source 
electrolyte) was varied: $\mathrm{KCl}, \mathrm{NaCl}$, and $\mathrm{LiCl}$; further the influence of the co-ion in the target electrolyte was studied: $\mathrm{KCl}, \mathrm{KClO}_{4}, \mathrm{KC}_{2} \mathrm{H}_{3} \mathrm{O}_{2}$; and finally, the effect of the concentration of the source electrolyte was determined: $\mathrm{KCl}$ $10 \mathrm{mM}, 100 \mathrm{mM}$, and $1 \mathrm{M}$. The last $100 \mathrm{~s}$ of the steady state currents at $2 \mathrm{~V}$ driving voltage were used to calculate the conductivity. All conductivities were normalized to the behavior of $100 \mathrm{mM} \mathrm{KCl}$ transport. The OEIPs have ohmic behavior within the voltage windows relevant to this work. The OPV component was characterized by measuring $\mathrm{I}(V)$ curves, illuminating with a $630 \mathrm{~nm} 3 \mathrm{~W}$ LED covered with a diffuser to provide uniform intensity across the devices (Edmund Optics $100 \mathrm{~mm}$ opal diffuser) and/or with a highdensity LED light source $\left(626 \mathrm{~nm}, 2.48 \mathrm{~mW} / \mathrm{cm}^{2}\right.$ with the diffuser). The OEIP part was characterized by measuring the ionic currents between source and target by immersing $\mathrm{Ag} / \mathrm{AgCl}$ electrodes into the reservoirs. The spectral response of the OPVs was measured using LEDs with peak emission at 630 , 660, 680, and $720 \mathrm{~nm}$ (Roithner LaserTechnik GmbH) operated at a suitable current so as to have an equal output light intensity of $2.6 \mathrm{~mW} / \mathrm{cm}^{2}$.

\section{Photodriven ion transport validation}

The integrated device function was tested by irradiation of the OPV/OEIP with a high-density LED light source $\left(626 \mathrm{~nm}, 2.48 \mathrm{~mW} / \mathrm{cm}^{2}\right.$ with a diffuser) and following the $\mathrm{H}^{+}$transport through the polyanion channel with a $\mathrm{pH}$ indicator. The source reservoir contained $0.1 \mathrm{M} \mathrm{HCl}$, the target was filled with $0.2 \mathrm{mM}$ MR hydrochloride in $0.1 \mathrm{M} \mathrm{KCl}$ at pH 7. The demonstration of larger ion pumping was done with $\mathrm{ACh}$ and the tandem series devices. The source was filled with $\mathrm{ACh}$ chloride $(\mathrm{AChCl}, 0.1 \mathrm{M})$ and the target with $0.1 \mathrm{M}$ $\mathrm{KCl}$. The wafer was illuminated for $2 \mathrm{~h}$ to ensure complete ion exchange from $\mathrm{H}^{+}$to $\mathrm{ACh}^{+}$. The wafer was then irradiated for intervals between 5 and $45 \mathrm{~min}$ and the target was collected, with a washing step between each. The amount of ACh was quantified with an acetylcholine esterase (AE)/choline oxidase/horseradish peroxidase/tetramethylbenzidine assay using a 96-well plate and a plate reader (Synergy H1, BioTek). The efficiency of pumping was determined using the altered series tandem devices that allowed online measurement of current with a Keithley 2602A source meter (Supplementary Fig. 5). The efficiency was calculated according to the following equation:

$\eta=\frac{n_{\text {Ach delivered }}}{Q \cdot F}$

where $\eta$ is the efficiency, $Q$ is the measured charge gone through the circuit, and $F$ is the Faraday constant. The same kind of devices were used for studying the online ion exchange and the different regimes of the device operation. During the exchange of the source electrolyte, the probes were disconnected.

\section{Integrated OEIP/OPV on-skin demonstration}

Through-finger operation was studied. The peeled device was placed onto a finger with a droplet of water for sufficient adhesion. The finger was put on a single $630 \mathrm{~nm}$ LED, the light intensity coming through the finger was $\sim 2 \mathrm{~mW} /$ $\mathrm{cm}^{2}$. The I $(V)$ characteristic of the OPV was measured in this configuration using standard microprobes. Then the device was preloaded with $\mathrm{H}^{+}$by running the OEIP independently using a current-voltage source. Afterwards, the device was placed on the finger again, the source was filled with $0.1 \mathrm{M}$ $\mathrm{HCl}$ and the target with $0.1 \mathrm{mM} \mathrm{MR}$ in $0.1 \mathrm{M} \mathrm{KCl}$ at $\mathrm{pH}$ 7. The device was irradiated through the finger and images were taken after 1, 2, $3 \mathrm{~min}$.

\section{Stability}

Devices from the same wafer were placed into a phosphate buffer saline solution. After 15 and 72 days ( 15 and 34 days in case of tandem devices), the OEIP functionality was tested. Afterwards, the devices were washed with DI, dried and the OPV characteristics were measured for both dry and PBS-treated devices. The values of $V_{O C} I_{S C}$ and MPP were normalized to the values of the same devices measured as fabricated. The devices were then tested for light-driven $\mathrm{H}^{+}$pumping as stated above.

\section{Flexibility}

$\mathrm{I}(V)$ curves of the peeled devices were measured after each bending cycle. The devices were bent over syringe needles $(\varnothing 1.2$ and $0.6 \mathrm{~mm}$ ) ensuring that each individual OPV in the series was exposed to the stress. The bending was repeated multiple times using each needle. Finally, the devices were crumbled and the I $(V)$ was measured again. The high-density LED array equipped with diffusor was used as the light source.

\section{DATA AVAILABILITY}

The datasets generated during and/or analysed during the current study are available from the corresponding authors on reasonable request.

\section{ACKNOWLEDGEMENTS}

The authors are grateful to the Knut and Alice Wallenberg foundation for support especially within the framework of the Wallenberg Centre for Molecular Medicine (WCMM) at Linköping University; the Swedish Foundation for Strategic Research (SSF), and the Swedish Research Council (Vetenskapsrådet, 2018-04505). We are grateful to Gustav Knutson for support in printing photolithography masks. Open access funding provided by Linköping University.

\section{AUTHOR CONTRIBUTIONS}

M.J., E.D.G., M.B. and D.T.S. conceived the research idea. D.T.S., M.B. and E.D.G. supervised and coordinated the research. M.J. fabricated and tested all devices, with fabrication and measurement assistance from T.A.S., D.P. and V.D. M.J. wrote the paper with input from all authors.

\section{ADDITIONAL INFORMATION}

Supplementary information accompanies the paper on the npj Flexible Electronics website (https://doi.org/10.1038/s41528-019-0060-6).

Competing interests: The authors declare no competing interests.

Publisher's note Springer Nature remains neutral with regard to jurisdictional claims in published maps and institutional affiliations.

\section{REFERENCES}

1. Katz, E. (ed.) Implantable Bioelectronics (Wiley-VCH, Weinheim, 2014).

2. Carrara, S. \& Iniewski, K. (eds) Handbook of Bioelectronics (Cambridge University Press, Cambridge, 2015).

3. Simon, D. T., Gabrielsson, E. O., Tybrandt, K. \& Berggren, M. Organic bioelectronics: bridging the signaling gap between biology and technology. Chem. Rev. 116, 13009-13041 (2016).

4. García Núñez, C., Manjakkal, L. \& Dahiya, R. Energy autonomous electronic skin npj Flex. Electron. 3, 1 (2019).

5. Kaltenbrunner, M. et al. An ultra-lightweight design for imperceptible plastic electronics. Nature 499, 458-463 (2013).

6. Isaksson, J. et al. Electronic control of $\mathrm{Ca}^{2+}$ signalling in neuronal cells using an organic electronic ion pump. Nat. Mater. 6, 673-679 (2007)

7. Sjöström, T. A. et al. A decade of iontronic delivery devices. Adv. Mater. Technol. 3, 1700360 (2018).

8. Simon, D. T. et al. Organic electronics for precise delivery of neurotransmitters to modulate mammalian sensory function. Nat. Mater. 8, 742-746 (2009).

9. Proctor, C. M. et al. Electrophoretic drug delivery for seizure control. Sci. Adv. 4 eaau1291 (2018).

10. Liu, S. et al. Conjugated polymer for voltage-controlled release of molecules. Adv. Mater. 29, 1701733 (2017).

11. Hwang, G. et al. Self-powered deep brain stimulation via a flexible PIMNT energy harvester. Energy Environ. Sci. 8, 2677-2684 (2015).

12. Amar, A. Ben, Kouki, A. B. \& Cao, H. Power approaches for implantable medical devices. Sensors 15, 28889-28914 (2015).

13. Park, S. et al. Self-powered ultra-flexible electronics via nano-grating-patterned organic photovoltaics. Nature 561, 516-521 (2018)

14. Moon, E., Blaauw, D. \& Phillips, J. D. Subcutaneous photovoltaic infrared energy harvesting for bio-implantable devices. IEEE Trans. Electron Devices 64, 2432-2437 (2017).

15. Haeberlin, A. et al. Successful pacing using a batteryless sunlightpowered pacemaker. Europace 16, 1534-1539 (2014).

16. Haeberlin, A. et al. The first batteryless, solar-powered cardiac pacemaker. Heart. Rhythm 12, 1317-1323 (2015)

17. Song, K. et al. Subdermal flexible solar cell arrays for powering medical electronic implants. Adv. Healthc. Mater. 5, 1572-1580 (2016).

18. Lu, L. et al. Biodegradable monocrystalline silicon photovoltaic microcells as power supplies for transient biomedical implants. Adv. Energy Mater. 8, 1-8 (2018).

19. Hassler, C., Boretius, T. \& Stieglitz, T. Polymers for neural implants. J. Polym. Sci. Part B Polym. Phys. 49, 18-33 (2011). 
20. Fortin, J. B. \& Lu, T.-M. Chemical Vapor Polymerization, The Growth and Properties of Parylene. (Springer Science+Business Media, New York, 2004).

21. Rand, D. et al. Direct electrical neurostimulation with organic pigment photocapacitors. Adv. Mater. 30, 1707292 (2018).

22. Poxson, D. J. et al. Capillary-fiber based electrophoretic delivery device. ACS Appl. Mater. Interfaces 11, 14200-14207 (2019).

23. Tybrandt, K. et al. Translating electronic currents to precise acetylcholine-induced neuronal signaling using an organic electrophoretic delivery device. Adv. Mater. 21, 4442-4446 (2009).

24. Jonsson, A. et al. Therapy using implanted organic bioelectronics. Sci. Adv. 1, e1500039 (2015).

25. Arbring Sjöström, T. et al. A crosslinked polyelectrolyte for improved selectivity and processability of iontronic systems. ACS Appl. Mater. Interfaces 9, 30247-30252 (2017)
Open Access This article is licensed under a Creative Commons Attribution 4.0 International License, which permits use, sharing, adaptation, distribution and reproduction in any medium or format, as long as you give appropriate credit to the original author(s) and the source, provide a link to the Creative Commons license, and indicate if changes were made. The images or other third party material in this article are included in the article's Creative Commons license, unless indicated otherwise in a credit line to the material. If material is not included in the article's Creative Commons license and your intended use is not permitted by statutory regulation or exceeds the permitted use, you will need to obtain permission directly from the copyright holder. To view a copy of this license, visit http://creativecommons. org/licenses/by/4.0/.

(c) The Author(s) 2019 